\title{
ESTUDO SOBRE A SIMETRIA TEMPORAL E SUAS IMPLICAÇÕES
}

1. Bassem Youssef Makhoul Junior, Graduando em bacharelado em Física, Universidade Estadual de Feira de Santana, e-mail: bassem ymj@hotmail.com

2. Ana Carla Peixoto Bitencourt, Departamento de Física, Universidade Estadual de Feira de Santana, e-mail: ana.bitencourt@gmail.com

3.Carlos Alberto Lima Riberio, Departamento de Física, Universidade Estadual de Feira de Santana, e-mail: $\underline{\text { carl@uefs.br }}$

\section{PALAVRAS-CHAVE: SIMETRIA TEMPORAL, AHARONOV-BOHM, GRAFENO INTRODUÇÃO}

O principal objetivo deste trabalho é ter uma noção conceitual, bem como a aquisição de ferramentas matemáticas sobre alguns efeitos quânticos e simetrias presentes em materiais de baixa dimensionalidade, com foco na simetria de inversão temporal. Para que no próximo ano de pesquisa, seja possível aprofundar mais sobre os anéis de grafeno (AHARONOV-BOHM,1959).

O grafeno é um material formado por uma monocamada de átomos de carbono arranjados de forma hexagonal. Sua descoberta, ou síntese, produziu um excelente cenário para testar várias teorias da Física de Partículas e Campos e de Fenômenos de Altas Energias. Diversos trabalhos foram publicados relacionando o efeito AharonovBohm com o Grafeno. Recentemente no sistema de anéis de grafeno foi também observado. O efeito Aharonov-Bohm é um ótimo exemplo de versatilidade na física. É um efeito de interferência quântica de natureza topológica.

Com o impacto do grafeno, industrias ao redor do mundo começaram a usar o método da fita adesiva de Geim, um dos ganhadores do Nobel de Física em 2010, para a produção de camadas de grafeno. O governo britânico investiu cerca de seis milhões de dólares, para a criação do instituto nacional de grafeno. Com o objetivo de competir com os maiores possuidores de patentes do grafeno, que são: China, Coreia e Estados Unidos. Podemos citar também duas grandes empresas de tecnologia que apresentam altas expectativas para grafeno, a Samsung e a I.B.M. (COLAPINTO,2014).

\section{MATERIAL E MÉTODOS OU METODOLOGIA}

Foram realizadas leituras críticas de artigos e livros que formaram a base do trabalho feito durante o ano de pesquisa. Após os estudos e análises, foram feitos textos críticos sobre o conteúdo que posteriormente eram discutidos com o orientador, visando solucionar duvidas e ideias que surgiram após a leitura do texto.

Inicialmente foi feito o levantamento bibliográfico sobre o tema, visando entender como as Simetrias da Mecânica Clássica atuam em sistemas físicos. Em seguida foram realizados estudos sobre o formalismo da Mecânica Analítica, especificamente o estudo da Equação de Euler-Lagrange e a Equação de Hamilton, para posteriormente aplicá-los no Teorema de Noether que relaciona simetrias e leis de conservação. Fez-se um levantamento bibliográfico sobre o grafeno, visando entender suas principais propriedades. Com isso, começamos a estudar o efeito Aharonov-Bohm e suas possíveis aplicações no grafeno. E por fim, para que houvesse um estudo sobre a simetria de inversão temporal, foi necessário o conhecimento da notação de Dirac que rege a mecânica quântica.

\section{RESULTADOS E/OU DISCUSSÃO}

O Cálculo Variacional tem como objetivo determinar extremos, ou seja, máximos ou mínimos de funcionais. Um funcional é uma função real cujo domínio é um espaço de funções. Então, um funcional associa um número real a cada função de uma certa classe de funções para as quais o funcional está definido.

Um exemplo de funcional: suponhamos dois pontos distintos num plano $\left(x_{1}, y_{1}\right)$ e $\left(x_{2}, y_{2}\right)$, e seja $Y(X)$ uma curva que ligando esses dois pontos. $O$ comprimento do arco infinitesimal no plano é dado por: 


$$
d S^{2}=d X^{2}+d Y^{2}
$$

ou

podemos ainda escrever como

$$
d S=\sqrt{1+\left(\frac{d Y}{d X}\right)^{2}} d x
$$

$$
S[Y]=\int_{x_{1}}^{x_{2}} \sqrt{1+(\dot{Y}(X))^{2}} d X .
$$

O comprimento do arco S é um funcional de $\mathrm{Y}$, isto é, a cada função diferenciável de há um valor real correspondente de $S[Y]$.

A Partir desta ideia foi possível encontrar a equação de Euler:

$$
\frac{\partial F}{\partial \bar{Y}}-\frac{d}{d X} \frac{\partial F}{\partial \dot{\bar{Y}}}=0
$$

As coordenadas necessárias para descrever a posição de uma ou mais partículas não precisam ser necessariamente as utilizadas geralmente, as cartesianas $(\mathrm{x}, \mathrm{y}, \mathrm{z})$. Quaisquer quantidades $q_{1}, q_{2}, \ldots, q_{n}$ que possam descrever as posições das partículas no sistema, sendo " $n$ " o grau de liberdade, servem e podem ser chamadas de coordenadas generalizadas. Os vínculos podem ser entendidos como limitações impostas sobre o movimento de um corpo, um exemplo, é o mover-se em uma reta(THORNTOM \& MARION,2004).

O Lagrangeano é definido como:

$$
\begin{gathered}
L=T-U, \\
L=f(q, \dot{q}, t),
\end{gathered}
$$

sendo $T$ a energia cinética e $U$ a energia potencial, e uma função que depende das posições generalizadas, velocidades generalizadas e tempo.

Usando a equação de Euler (04) e a definição do Lagrangeano teremos:

$$
\frac{\partial L}{\partial q}-\frac{d}{d t} \frac{\partial L}{\partial \dot{q}}=0 .
$$

Para se encontrar o Hamiltoniano, basta tomar o diferencial total do Lagrangeano em função do tempo e admitir que o tempo se passe de maneira homogênea. Então:

$$
\frac{d L}{d t}=\sum \frac{\partial L}{\partial q} \dot{q}+\sum \frac{\partial L}{\partial \dot{q}} \ddot{q}+\frac{\partial L}{\partial t}
$$

usando a equação (07), e arrumando toda equação, obteremos:

$$
H=\sum p \dot{q}-L_{s}
$$

a equação (09) é dita como Hamiltoniano do sistema, e representa a energia total do desse, desde que não haja dependência explicita do tempo.

O teorema de Noether diz que para cada lei de conservação, existe uma simetria relacionada. A dedução feita acima do Hamiltoniano é um exemplo disto, para conservação da energia existe uma simetria por trás, e esta é a homogeneidade temporal (LEMOS,2007).

O teorema também tem uma forma algébrica, definida da seguinte forma:

$$
C=\sum \frac{\partial L}{\partial \dot{q}}(\dot{q} X-\varphi)-L X
$$

O efeito Aharonov-Bohm demonstra que existe uma influência do vetor potencial na teoria Quântica. A partir de um aparato experimental, se é confinado um campo magneto dentro de um solenoide, de modo que, fora desse não exista influência 
de campo magnético. Quando um feixe de elétrons é divido em 2 e ambos passam ao redor do solenoide, ao se juntarem foi observado um padrão de interferência, portanto, uma diferença de fase, algo que não deveria acontecer (AHARONOV-BOHM,1959). A única explicação é que o campo magnético que está confinado dentro do solenoide, de alguma forma conseguiu alterar a fase do elétron. O Hamiltoniano do sistema será então:

$$
H=\frac{1}{2 m}\left(\vec{p}-\frac{q}{c} \vec{A}\right)^{2}+q \phi .
$$

No outono de 2002, André Geim pediu para um de seus estudantes de Ph.D., Da Jiang, a tarefa de isolar amostras extremamente finas de camadas de carbono. Algumas semanas depois, Jiang entregou uma única partícula de carbono em uma placa de petri, que nada mais é que um recipiente cilíndrico, achatado, de vidro ou plástico que os profissionais de laboratório utilizam para realizar analise de de microorganismos.. Geim, insatisfeito com os resultados, pediu para que ele recomeçasse, mas Jiang já havia usado toda a amostra que ele tinha. Foi então que Geim percebeu, em uma das lixeiras de seus próprios colegas, uma bola de fita adesiva cinza e levemente brilhante, com um filme de grafite colado nela. Após pôr a fita sob um microscópio, Geim descobriu que as camadas de grafite eram menores do que qualquer outra que ele já tinha visto. Dobrando a fita e apertando as duas partes entre si, ele foi capaz de fazer camadas ainda mais finas. Assim, Geim criou o primeiro material de duas dimensões. Físicos teóricos já tinham especulado a existência de tal material e o chamavam de grafeno (NOVOSELOV,2011).

Um vetor representado no espaço de Dirac é descrito como ket e tem a seguinte forma:

$$
|A\rangle \text {, }
$$

uma componente do dual desse espaço é denominada bra, e tem a seguinte forma:

$$
\langle B| .
$$

O produto escalar de um bra ket é definido como

$$
\langle A \mid B\rangle \text {. }
$$

Algumas propriedades básicas são:

$$
\begin{gathered}
\sum_{n}|n\rangle\langle n|=1, \\
\int d \vec{x}|\vec{x}\rangle\langle\vec{x}| .=1 .
\end{gathered}
$$

A Simetria de Inversão Temporal me diz que quando pararmos um sistema em movimento em certo ponto, e o fazer retroceder, o sistema começará a evoluir exatamente na mesma trajetória em que começou a se mover. $\mathrm{E}$ as leis que regem seu movimento de ida e volta devem ser as mesmas (SHANKAR,2014).

Podemos definir:

$$
\Theta=\mathrm{UK} \text {, }
$$

sendo $U$ um operador antiunitário e $K$ é o operador complexo conjugado, que toma o conjugado de qualquer variável que acompanha que multiplique o ket.

O operador de inversão temporal também é um operador antiunitário, assim:

$$
|\psi\rangle \rightarrow \Theta|\psi\rangle,
$$

sendo $\Theta|\psi\rangle$ o estado de inversão temporal do $|\psi\rangle$.

Vamos agora considerar um operador linear $\hat{A}$, o seu transposto $\hat{A}^{T}$ e os estados $|\psi\rangle$ e $|\varphi\rangle$, sendo

$$
\begin{aligned}
& |\tilde{\psi}\rangle=\Theta|\psi\rangle, \\
& |\tilde{\varphi}\rangle=\Theta|\varphi\rangle,
\end{aligned}
$$


então

A prova da equação (79)

$$
\langle\varphi|\hat{A}| \psi\rangle=\left\langle\tilde{\psi}\left|\Theta \hat{A} \Theta^{-1}\right| \tilde{\varphi}\right\rangle .
$$

$$
\begin{gathered}
|\gamma\rangle=\hat{A}^{T}|\varphi\rangle, \\
\langle\gamma|=\langle\varphi| \hat{A}, \\
\langle\varphi|\hat{A}| \psi\rangle=\langle\gamma \mid \psi\rangle=\langle\tilde{\gamma} \mid \tilde{\psi}\rangle, \\
\left\langle\tilde{\psi}\left|\Theta \hat{A}^{T}\right| \varphi\right\rangle=\left\langle\tilde{\psi}\left|\Theta \hat{A}^{T} \Theta^{-1} \Theta\right| \varphi\right\rangle=\left\langle\tilde{\psi}\left|\Theta \hat{A}^{T} \Theta^{-1}\right| \tilde{\varphi}\right\rangle .
\end{gathered}
$$

Podemos então definir:

$$
\Theta \hat{A} \Theta^{-1}= \pm \hat{A},
$$

então

$$
\langle\psi|\hat{A}| \psi\rangle=\langle\tilde{\psi}|\hat{A}| \tilde{\psi}\rangle,
$$

sendo $\langle\tilde{\psi}|\hat{A}| \widetilde{\psi}\rangle$ o valor esperado calculado com respeito ao estado reverso no tempo.

\section{CONSIDERAÇÕES FINAIS}

Portanto, este último ano de pesquisa proporcionou um aprendizado muito extenso, vimos através do teorema de Noether como as simetrias aplicadas no Lagrangeano conseguem me fornecer entidades muito importantes, tais como a equação (09), sendo uma delas a energia total do sistema físico, que vale para qualquer sistema, seja Clássico ou Quântico. O efeito Aharonov-Bohm mostrou a importância de vetor potencial na Mecânica Quântica, e como uma grandeza clássica que é campo magnético, alterou uma medida quântica que é a energia total do elétron. A partir do levantamento bibliográfico sobre o grafeno pode-se perceber que ele tem o potencial de se tornar o futuro nas novas tecnologias, seu conjunto de características faz com que ele seja o material ideal para diversas aplicações, tais como: paneis solares, filmes finos, baterias, supercapacitores etc. Com a análise matemática da Simetria de Inversão Temporal conseguimos deduzir uma propriedade muito importante, que é a equação (23) que será de grande importância na hora de descrever o mapeamento de uma partícula em um anel de grafeno. Finalizando, todo esse trabalho teve como meta principal, fornecer as ferramentas matemáticas e físicas para que seja possível abordar o estudo dos anéis de grafeno a partir da equação de Dirac que é relativística.

\section{REFERÊNCIAS}

Y. Aharonov and D. Bohm, Significance of Electromagnetic Potentials in the Quantum Theory Phys. Rev., 115, 485, (1959)

R. Shankar, Principles of Quantum Mechanics, New Haven Connecticut, Yale university, Plenum Publishers, 1994

Thornton, S. T. e Marion, J. B., Dinâmica Clássica de Partículas e Sistemas, Editora Cengage, 5. $^{\circ}$ Edição, 2004.

Nivaldo A. Lemos. Mecânica analítica. São Paulo Livraria da Física, 2007

K. S. Novoselov, Nobel Lectures, Graphene: Materials in the Flatland, 2011, page (6986-7002).

J.J. Sakurai, Jim Napolitano, Mecânica Quântica Moderna, Bookman, $2^{\circ}$ edição, Porto Alegre, 2013

Wencai Ren and Hui-Ming Cheng, NATURE, The global growth of graphene, vol.9, October 2014, page (726-730).

John Colapinto, The New Yorker, Graphene may be the most remarkable substance ever discovered. But what it's for?, New York, December 22, 2014. 